\title{
Exposition du cristallin du praticien lors des traitements avec des rayons $X$ de basse énergie
}

\author{
S. Marcié^, C. Dejean, J. Feuillade, M. Gautier, K. Benézery, M.E. Chand, A. Courdi et J.P. Gérard \\ Centre Antoine-Lacassagne, 06100 Nice, France.
}

Reçu le 12 juin 2014 - Accepté le 30 juillet 2014

\begin{abstract}
Résumé - Les traitements utilisant des rayons X de basse énergie continuent à être réalisés mais en faible nombre. Le praticien se trouve en général près du patient pour maintenir l'appareil et expose donc ses yeux. Avec la modification envisagée de la limite maximale d'exposition du cristallin, des mesures ont été jugées nécessaires pour mieux en connaître l'exposition. Pour cela une simulation des traitements a été réalisée. Pour la dose d'un traitement superficiel de 42 Gy, l'exposition du cristallin derrière des verres radio atténuateurs se situe entre 130 et $43 \mu \mathrm{Sv}$ pour des distances entre 40 et $60 \mathrm{~cm}$. Pour l'intra cavitaire les valeurs obtenues sont vingt fois plus faibles. L'atténuation par les verres est supérieure à $90 \%$. Le port des lunettes avec verres radio atténuateurs permet de minimiser la dose au cristallin de façon significative. L'application de la nouvelle limite maximale n' aura pas d'impact sur le nombre de traitements.
\end{abstract}

\begin{abstract}
Exposure of the practitioner's lens during treatment with low-energy X-rays. Treatment using lowenergy X-rays is still carried out but in small numbers. The practitioner is usually near the patient to hold the device and thus exposes his eyes. With the proposed change to the maximum limit, measurement is necessary to understand the exposure of the lens better. For this, a treatment simulation was performed. For the dose of a surface treatment of $42 \mathrm{~Gy}$, exposure to the lens, with protective eyewear, is between 130 and $43 \mu \mathrm{Sv}$ for distances between 40 and $60 \mathrm{~cm}$. For intra-cavitary use the values obtained are twenty times lower. The attenuation of the protective eyewear is greater than $90 \%$. Protective eyewear significantly minimises the dose to the lens. The application of the new maximum limit will not impact on the number of treatments.
\end{abstract}

Keywords: low-energy X-radiation / radiotherapy / lens

\section{Introduction}

Les traitements de radiothérapie par des $\mathrm{X}$ de basse énergie $(<200 \mathrm{kV})$ sont réalisés depuis longtemps et continuent à l'être même si leur nombre a énormément chuté au cours de ces vingt dernières années. Le développement de nouveaux appareils comme le «Papillon50 ${ }^{\mathrm{TM}}$ » (Gérard et al., 2011) a amené un regain d'intérêt pour ces traitements.

Pour une réalisation précise de l'irradiation, il est souvent nécessaire que le praticien maintienne l'appareil au contact même de la cible (par exemple pour une paupière), et s'assure visuellement de la position de l'applicateur sur la zone à traiter. Cela implique une exposition des yeux qui sont protégés par des lunettes à verres radio atténuateurs.

La modification envisagée de la limite maximale pour le cristallin, passage de 150 à $20 \mathrm{mSv}$ (Directive 2013/59/Euratom (2013)), nous a amené à essayer de déterminer plus précisément le niveau d'exposition du cristallin du praticien.

\footnotetext{
^ serge.marcie@nice.unicancer.fr
}

\section{Matériels}

L'appareil de traitement (Fig. 1) est le «Papillon50 ${ }^{\mathrm{TM}}$ » (Gérard et al., 2012). Les rayons X émis ont une énergie maximale de $50 \mathrm{keV}$ et une énergie moyenne de $37 \mathrm{keV}$ (Croce et al., 2012). Des applicateurs (Fig. 2) sont ajoutés pour les traitements, de diamètres 10 à $30 \mathrm{~mm}$ avec des DSP de 3 à $4 \mathrm{~cm}$. Le pupitre se situe à environ 2 mètres de la zone à traiter, dans la salle de traitement.

Un tablier, des gants et une paire de lunettes à verres radio atténuateurs sont portés par le praticien qui effectue le traitement.

Pour les mesures, des détecteurs Babyline 91 et Victoreen (modèle 451) sont utilisés, sans capuchon, pour les mesures sans lunettes. Des dosimétres thermoluminescents (diamètre $5 \mathrm{~mm}$ et épaisseur $1 \mathrm{~mm}$ ) GR200A (Bacci et al., 1993 ; SFPM, 2000), positionnés en surface dans des pochettes en vinyle (3 par pochette), sont utilisés pour les mesures avec et sans lunettes. La lecture est faite avec un lecteur Harshaw 5500. Les dosimétres sont étalonnés avec un rayonnement de photons de $6 \mathrm{MV}$ et pour 10 unités moniteur à la distance conventionnelle de $1 \mathrm{~m}$ soit une dose de $11 \mathrm{cGy}$. 


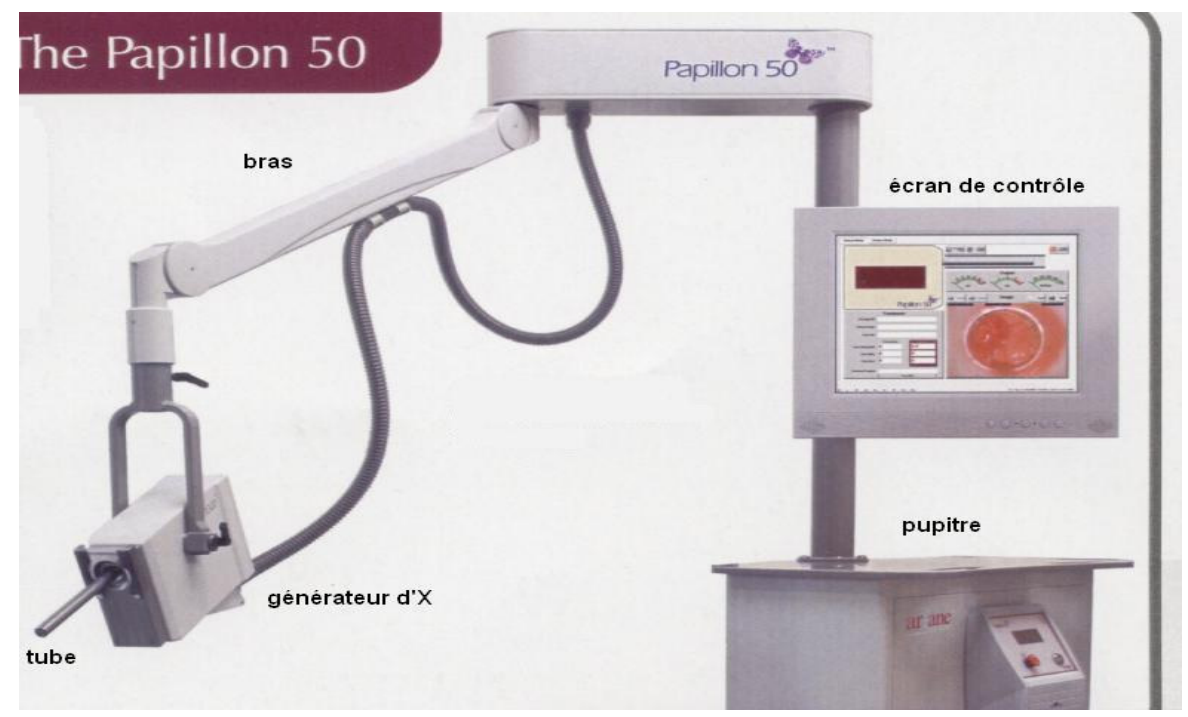

Fig. 1. L'appareil Papillon50.

The Papillon50 device.

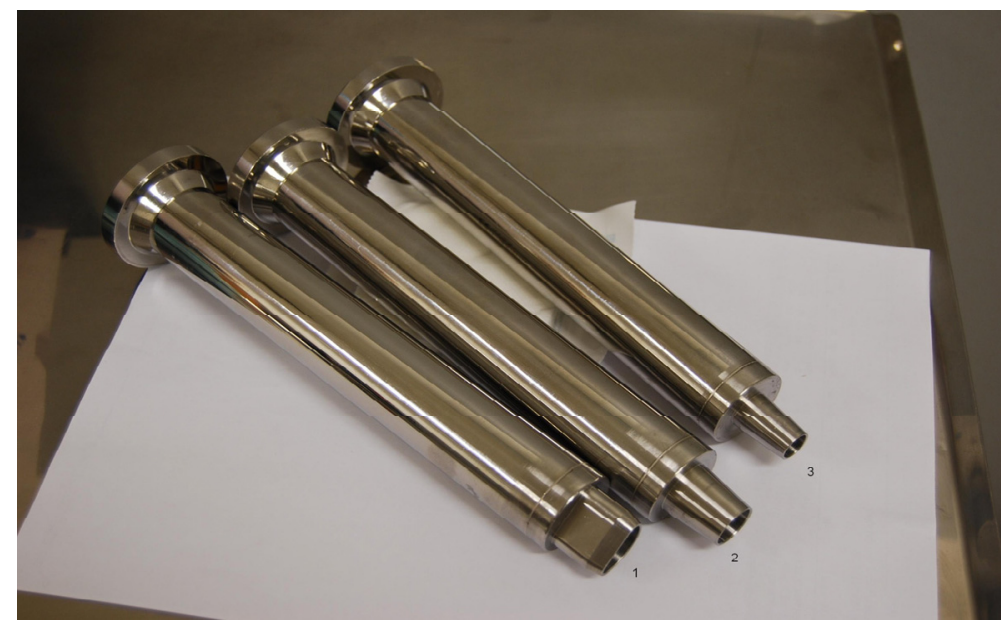

Fig. 2. Quelques applicateurs ( 1 : pour la paupière ; 2 : diamètre $15 \mathrm{~mm} ; 3$ : diamètre $10 \mathrm{~mm}$ ). Some applicators (1: for the eyelid; 2: $15 \mathrm{~mm}$ diameter; $3: 10 \mathrm{~mm}$ diameter).

La tête d'un fantôme anthropomorphe Rando-Alderson (Fig. 3) simule le praticien tandis qu'un ensemble de plaques de plexiglass simule le patient.

\section{Méthodes}

\subsection{Les traitements}

Pour les localisations superficielles (peaux, paupières) le praticien doit maintenir l'applicateur au contact de la lésion et surveiller visuellement. Les doses séances sont de 7 Gy et il y a 5 ou 6 séances étalées sur 3 à 4 semaines.

Pour la localisation intra cavitaire (rectum) le praticien maintient l'applicateur mais n'a pas besoin de surveillance visuelle et peut donc détourner son regard. Il se positionne naturellement plus en arrière de l'appareil. Les doses par séances sont variables, échelonnées entre 35 et 15 Gy et il y a 3 ou 4 séances étalées sur 3 à 6 semaines.
Pour les 3 années qui viennent de s'écouler la moyenne des séances par an est de 136 pour les localisations superficielles et 35 pour l'intra cavitaire.

\subsection{Mesures de simulation}

Les détecteurs sont positionnés à différentes distances pour simuler le positionnement du praticien entre 40 et $60 \mathrm{~cm}$ de la source (zone dans laquelle va se situer l'œil du praticien en fonction de sa taille et de la localisation à traiter).

Pour évaluer l'atténuation des lunettes les dosimétres thermoluminescents sont positionnés au même niveau que les détecteurs avec et sans lunettes soit au niveau de l'œil du fantôme qui simule le praticien, sur le côté de l'appareil comme représenté sur la figure 3.

Les irradiations sont faites pour 30 Gy délivrés en surface car il est impossible de faire des mesures avec une dose importante, l'appareil s'arrêtant si la température du liquide de 


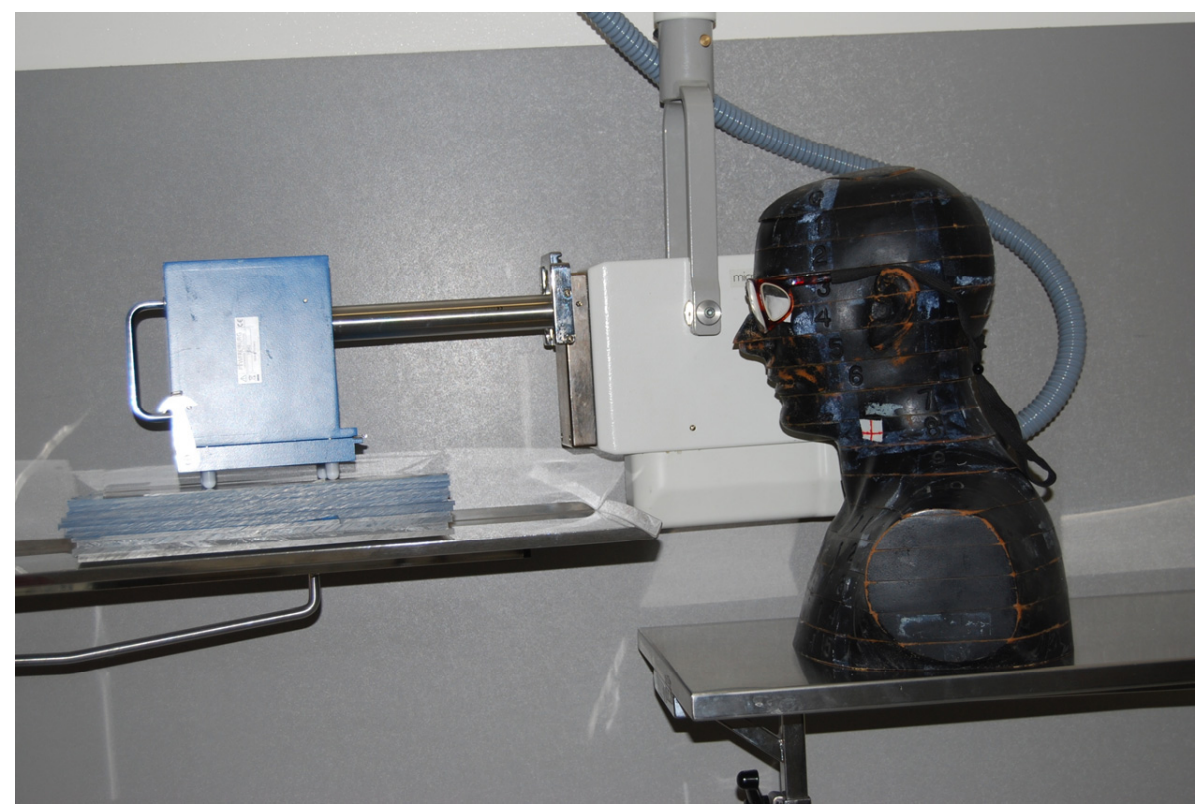

Fig. 3. Photographie de la simulation.

Picture of the simulation.

Tableau 1. Valeurs en $\mu \mathrm{Sv}$ des expositions du cristallin protégé ou pas, pour différentes distances et pour les localisations superficielles (42 Gy) ou intra cavitaires (90 Gy).

$\mathrm{Sv}$ values of exposure with and without protective eyewear, for different distances and superficial (42 Gy) or intra-cavity (90 Gy) localisations.

\begin{tabular}{|c|c|c|c|c|}
\hline $\begin{array}{l}\text { Distances } \\
\text { en } \mathrm{cm}\end{array}$ & $\begin{array}{l}\text { Superficiel } \\
\text { sans verres }\end{array}$ & $\begin{array}{l}\text { Superficiel } \\
\text { avec verres }\end{array}$ & $\begin{array}{c}\text { Intra cavitaire } \\
\text { sans verres }\end{array}$ & $\begin{array}{l}\text { Intra cavitaire } \\
\text { avec verres }\end{array}$ \\
\hline 40 & 1470 & 130 & 90 & 8 \\
\hline 50 & 910 & 80 & 54 & 5 \\
\hline 60 & 633 & 43 & 42 & 3 \\
\hline
\end{tabular}

refroidissement est trop élevée. Les valeurs correspondantes à un traitement ont été obtenues en effectuant une règle de trois.

\section{Résultats et discussion}

Le tableau 1 donne les résultats des mesures de simulations des dosimétres thermoluminescents avec et sans les lunettes. Pour les mesures sans lunettes la valeur indiquée est la moyenne entre les moyennes des mesures avec le détecteur Victoreen et les détecteurs thermoluminescents car ces moyennes sont très proches (écart inférieur à $5 \%$ ). Les résultats avec la Babyline sont inférieurs de $10 \%$.

Pour les valeurs des applications intra cavitaires avec lunettes les valeurs sont calculées en utilisant la valeur mesurée sans lunettes et le coefficient d'atténuation des applications superficielles, les mesures par thermoluminescence n'étant pas significatives.

L'atténuation des verres est supérieure à $90 \%$. L'application intra cavitaire est beaucoup moins irradiante, la source de rayonnement étant à l'intérieur du patient.

Lors d'une irradiation exceptionnelle de radiothérapie peropératoire avec cet appareil (Marcié et al., 2014), les mesures au niveau du front étaient de 17 ou $30 \mu \mathrm{Sv}$ suivant l'instrument de mesure et pour 14 Gy délivrés dans la cavité opératoire. Ces valeurs sont à rapprocher de nos mesures, qui ramenées à $14 \mathrm{~Gy}$, donnent $14 \mu \mathrm{Sv}$.

Pour estimer la dose au cristallin lors d'un traitement, l'alternative proposée par Bordy (2014) est de mesurer la dose au niveau du thorax. Au préalable, une simulation permet de connaître le rapport entre la mesure au niveau du cristallin et celle du thorax.

Sur la base du nombre de patients traités par an, et si c'était le même praticien (ce qui n'est pas le cas car plusieurs médecins réalisent ces traitements), l'exposition superficielle du cristallin, avec le port des lunettes et pour la distance la plus proche, serait de :

- Pour les traitements des lésions superficielles : avec une moyenne de 23 patients par an, 42 Gy par patient, l'exposition annuelle est de $3 \mathrm{mSv}$.

- Pour les traitements intra cavitaires : avec une moyenne de 12 patients par an, une moyenne de 90 Gy par patient, l'exposition annuelle est de $1 \mathrm{mSv}$.

Avec le nombre de patients actuels, la nouvelle limite n'est pas dépassée. Il faudrait augmenter par 7 le nombre de patients en superficiel et par 20 celui des applications intra cavitaires.

\section{Conclusion}

La nouvelle norme concernant le cristallin va nécessiter une plus grande attention à la position du praticien particulièrement pour les localisations superficielles mais elle ne constitue pas une limitation du nombre de patients traités. C'est encore plus valable pour les traitements en intra cavitaire. Le port des lunettes et la distance doivent rester des règles de bonne pratique de ces traitements. 


\section{Références}

Bacci C. et al. (1993) Comprehensive study on $\mathrm{LiF}: \mathrm{Cu}, \mathrm{Mg}, \mathrm{P}$ (GR-200A), Radiat. Prot. Dosim. 47 215-218.

Bordy J.M. (2014) Proposition d'un critère d'aide pour le choix entre une évaluation directe ou indirecte de la dose au cristallin. Poster. Dans : $53^{e s}$ journées scientifiques de la SFPM, Deauville.

Croce O. et al. (2012) Contact radiotherapy using a $50 \mathrm{kV}$ X-ray system : evaluation of relative dose distribution with the Monte Carlo code Penelope and comparison with measurements, Radiat. Phys. Chem. 81 (6), 609-617.

Directive 2013/59/Euratom du Conseil du 5 décembre 2013 fixant les normes de base relatives à la protection sanitaire contre les dangers résultant de l'exposition aux rayonnements ionisants et abrogeant les directives 89/618/Euratom, 90/641/Euratom, 96/29/Euratom, 97/43/Euratom et 2003/122/Euratom.

Gérard J.P. et al. (2011) Renaissance of contact X-ray therapy for treating rectal cancer, Expert Rev. Med. Devices 8 (4), 483-482.

Gérard J.P. et al. (2012) Développement de l'appareil Papillon50 ${ }^{\mathrm{TM}}$ et de ses applicateurs pour la radiothérapie $50 \mathrm{kV}$ des cancers du rectum et de la peau, IRBM 33, 109-116.

Marcié S. et al. (2014) La radioprotection pour un cas exceptionnel de radiothérapie peropératoire dans un site non radioprotégé, Radioprotection 49 (2), 135-138.

SFPM (2000) Guide pour la mise en oeuvre en radiothérapie externe de l'assurance de qualité par mesures in vivo par dosimétres thermoluminescents et semi-conducteurs. Rapport SFPM $\mathrm{N}^{\circ} 18-2000$.

Cite this article as: S. Marcié, C. Dejean, J. Feuillade, M. Gautier, K. Benézery, M.E. Chand, A. Courdi, J.P. Gérard. Exposition du cristallin du praticien lors des traitements avec des rayons X de basse énergie. Radioprotection 49(4), 289-292 (2014). 